\title{
Machine learning assisted DSC-MRI radiomics as a tool for glioma classification by grade and mutation status
}

\author{
Carole H. Sudre ${ }^{1,2,3 \dagger}$, Jasmina Panovska-Griffiths ${ }^{4,5,6^{*}+} \mathbb{D}$, Eser Sanverdi ${ }^{7 \dagger}$, Sebastian Brandner ${ }^{8}$, \\ Vasileios K. Katsaros ${ }^{9,10}$, George Stranjalis ${ }^{10}$, Francesca B. Pizzini ${ }^{11}$, Claudio Ghimenton ${ }^{12}$, \\ Katarina Surlan-Popovic ${ }^{13,14}$, Jernej Avsenik ${ }^{13,14}$, Maria Vittoria Spampinato ${ }^{15}$, Mario Nigro ${ }^{15}$, \\ Arindam R. Chatterjee ${ }^{15}$, Arnaud Attye ${ }^{16}$, Sylvie Grand ${ }^{16}$, Alexandre Krainik ${ }^{16}$, Nicoletta Anzalone ${ }^{17}$ \\ Gian Marco Conte ${ }^{17}$, Valeria Romeo ${ }^{18}$, Lorenzo Ugga ${ }^{18}$, Andrea Elefante ${ }^{18}$, Elisa Francesca Ciceri ${ }^{12,18}$, \\ Elia Guadagno ${ }^{19}$, Eftychia Kapsalaki ${ }^{20}$, Diana Roettger ${ }^{21}$, Javier Gonzalez ${ }^{21}$, Timothé Boutelier $^{22}$, \\ M. Jorge Cardoso ${ }^{1,2,3}$ and Sotirios Bisdas ${ }^{7,23}$
}

\begin{abstract}
Background: Combining MRI techniques with machine learning methodology is rapidly gaining attention as a promising method for staging of brain gliomas. This study assesses the diagnostic value of such a framework applied to dynamic susceptibility contrast (DSC)-MRI in classifying treatment-naïve gliomas from a multi-center patients into WHO grades II-IV and across their isocitrate dehydrogenase (IDH) mutation status.
\end{abstract}

Methods: Three hundred thirty-three patients from 6 tertiary centres, diagnosed histologically and molecularly with primary gliomas $(\mathrm{IDH}$-mutant $=151$ or IDH-wildtype $=182)$ were retrospectively identified. Raw DSC-MRI data was post-processed for normalised leakage-corrected relative cerebral blood volume ( $\mathrm{rCBV}$ ) maps. Shape, intensity distribution (histogram) and rotational invariant Haralick texture features over the tumour mask were extracted. Differences in extracted features across glioma grades and mutation status were tested using the Wilcoxon twosample test. A random-forest algorithm was employed (2-fold cross-validation, 250 repeats) to predict grades or mutation status using the extracted features.

Results: Shape, distribution and texture features showed significant differences across mutation status. WHO grade II-III differentiation was mostly driven by shape features while texture and intensity feature were more relevant for the III-IV separation. Increased number of features became significant when differentiating grades further apart from one another. Gliomas were correctly stratified by mutation status in $71 \%$ and by grade in $53 \%$ of the cases ( $87 \%$ of the gliomas grades predicted with distance less than 1).

(Continued on next page)

\footnotetext{
*Correspondence: j.panovska-griffiths@ucl.ac.uk

${ }^{\dagger}$ Carole H. Sudre, Jasmina Panovska-Griffiths and Eser Sanverdi contributed equally to this work.

${ }^{4}$ Department of Applied Health Research, Institute of Epidemiology \& Health

Care, University College London, London, UK

5 Institute for Global Health, University College London, London, UK

Full list of author information is available at the end of the article
}

(c) The Author(s). 2020 Open Access This article is licensed under a Creative Commons Attribution 4.0 International License, which permits use, sharing, adaptation, distribution and reproduction in any medium or format, as long as you give appropriate credit to the original author(s) and the source, provide a link to the Creative Commons licence, and indicate if changes were made. The images or other third party material in this article are included in the article's Creative Commons licence, unless indicated otherwise in a credit line to the material. If material is not included in the article's Creative Commons licence and your intended use is not permitted by statutory regulation or exceeds the permitted use, you will need to obtain permission directly from the copyright holder. To view a copy of this licence, visit http://creativecommons.org/licenses/by/4.0/ The Creative Commons Public Domain Dedication waiver (http://creativecommons.org/publicdomain/zero/1.0/) applies to the data made available in this article, unless otherwise stated in a credit line to the data. 
(Continued from previous page)

Conclusions: Despite large heterogeneity in the multi-center dataset, machine learning assisted DSC-MRI radiomics hold potential to address the inherent variability and presents a promising approach for non-invasive glioma molecular subtyping and grading.

Keywords: Diagnostic machine learning, Glioma stratification, Isocitrate dehydrogenase; DSC-MRI

\section{Key points}

- On highly heterogenous, multi-centre data, machine learning on DSC-MRI features can correctly predict glioma IDH subtyping in $71 \%$ of cases and glioma grade II-IV in $53 \%$ of the cases $(87 \%<1$ grade difference).

- Shape features distinguish best grade II from grade III gliomas.

- Texture and distribution features distinguish best grade III from grade IV tumours.

\section{Importance of study}

This work illustrates the diagnostic value of combining machine learning and dynamic susceptibility contrastenhanced MRI (DSC-MRI) radiomics in classifying gliomas into WHO grades II-IV as well as across their isocitrate dehydrogenase (IDH) mutation status.

Despite the data heterogeneity inherent to the multicentre design of the studied cohort (333 subjects, 6 centres) that greatly increases the theoretical challenges of machine learning frameworks, good classification performance (accuracy of $53 \%$ across grades $(87 \%<1$ grade difference) and $71 \%$ across mutation status) was obtained. Therefore, our results provide a proof-of-concept for this emerging precision medicine field that has good generalisability and scalability properties. Introspection on the classification errors highlighted mostly borderline cases and helped underline the challenges of a categorical classification in a pathological continuum.

With its strong generalisability property, its ability to further incorporate participating centres and its possible use to identify borderline cases, the proposed machine learning framework has the potential to contribute to the clinical translation of machine-learning assisted diagnostic tools in neuro-oncology.

\section{Background}

The most recent grading system of gliomas has integrated the mutation status of key encoding genes prompting a ground-breaking change in the context of glioma diagnosis and treatment $[1,2]$ with further molecular stratification achieved by determining CDKN2A/ $B$ status [3]. Tumour anatomical features and gadolinium enhancement properties are not sufficient for this task and are extremely variable across different types and grades of gliomas with low specificity rates $[4,5]$. To overcome this limitation, advanced imaging techniques such as diffusion weighted imaging (DWI), MR spectroscopy, and dynamic susceptibility contrast (DSC)-MRI (also known as perfusion-weighted MRI) have been tested. Perfusion-weighted MRI provides biomarkers tightly linked to the tumour vascularity and thus indices of the biochemical and genetic substrates related to neo-angiogenesis [6, 7]. 'Gain-of-function' isocitrate dehydrogenase (IDH) mutations result in the accumulation of the "oncometabolite" 2-hydroxy glutarate (HG) and cause an increase in the activity of the hypoxia-inducible factor- 1 , which has a pivotal role in the energy metabolism, angiogenesis and apoptosis [8]. Therefore, perfusion MRI biomarkers carry the potential to act as diagnostic surrogates of IDH mutation status and further for tumour grading.

DSC-MRI is often favoured, compared to other MRIbased perfusion techniques, for its higher temporal resolution and sensitivity for detecting abnormal angiogenesis resulting in high accuracy rates for baseline tumour grading and tumour surveillance [7]. To date, these encouraging findings have been mostly obtained in single-institution settings utilising different DSC-MRI acquisition techniques and post-processing software packages for quantitative analysis. This can result in significant variation [7] and, hence, limit the diagnostic value of the technique impeding its large-scale application for non-invasive tumour phenotyping [9-11]. Recently, a consensus paper for DSC-MRI acquisition guidelines aimed to act as a blueprint and provide a framework for achieving routine success with this technique [12].

The vast majority of the published DSC-MRI studies have focused on establishing the value of the tumour region of interest rather than encompassing the whole tumour. They have made use of histogram analyses, which nonetheless reflect numeric values of the voxels and only help to calculate first-order statistics. By contrast to first-order statistics, second- and higher-order statistical analyses, which allow measures of not only local voxel-wise values but also incorporate neighbouring information, have been suggested as more reliable and elaborative methods for characterisation of the region-of-interest [13]. Texture analysis, as a contextual quantification method, has already embarked in the 
medical imaging literature as a method that can detect tissue heterogeneity and complexity [13, 14]. Radiomics, applied in the clinical context of glioblastoma, have generally been performed on anatomical sequences and applied to distinguish between GBM subtypes [15], for prediction of survival rates [16] and prognosis [17], prediction of response to treatment [18], as well as risk stratification [19]. Deep-learning based techniques have recently received a sustained attention due to their high performance in such classification tasks [20, 21]. Nonetheless, there is limited biological insight in their decision process making failure cases difficult to interpret. An alternative is to use the classical feature-based machine learning methods as diagnostic tool and to evaluate the relevance of acquisition sequences. In such a framework, interaction between clinical interpretation and machine learning is essential. Indeed, an iterative loop between clinical interpretation and machine learning analysis based on advanced features can help to define most distinguishing images properties and exclude non-relevant ones. Such process would result in the definition of disease specific imaging signatures.

While the high heterogeneity among DSC-MRI acquisition protocols may contribute to lowering the diagnostic value of any single first-order statistic, this work sought to examine the role of higher-order analysis and machine-learning assisted radiomics in mitigating these limitations and augment the diagnostic accuracy of DSC-MRI for tumour staging with emphasis on IDH molecular subtyping.

Specifically, this study aims to assess the accuracy of combining DSC-MRI radiomics with supervised machine learning algorithms in classifying gliomas by grade and mutation status using multi-centre dataset. Our hypothesis is that different combination of MRI features are to be used according to the distinctions to be looked after encompassing either tissue heterogeneity between high grade GBM or measures of dissemination (using shape assessment) at lower grades. Compared to existing work, including the most recent one by Lu et al. [22], where a publicly available database was used focusing solely on structural imaging, this work focuses for first time on the use of DSC-MRI acquisition in a multicentre setting. The heterogeneity inherent to the multi-centre quality of the data, makes the task of tumour and molecular phenotyping even more challenging. If successful, the proposed platform is readily expandable to include more participating institutions or utilising different machine learning approaches - all aiming to increase the diagnostic capacity and power to determine the most appropriate parameters for conducting DSC-MRI experiments. With this in mind, this work has potential to become a first step for a widespread application of machinelearning assisted diagnostic aid in neuro-oncology.

\section{Methods}

Different components of the methodology in this study are shown in Fig. 1a with the post-processing of the tumour segmentation and rCBV maps leading to feature extraction depicted in Fig. 1b.

\section{Study design and ethics}

This multi-center study was based on retrospectively identified or prospectively acquired neuro-oncology MRI data collection in the participating centres, according to the local institutional review boards guidelines and the respective local ethical committees' approvals. Data sharing agreements between the collaborators and the central reading and post-processing site were also in place.

\section{Subjects}

DSC-MRI data of 359 patients from 6 university hospitals with histopathologically and molecularly confirmed primary, treatment naïve gliomas (WHO grades II-IV) between January 2010 and May 2018 were consecutively collected and screened for this study. The inclusion criteria were as follows: i) existence of preoperative DSCMRI examination raw data suitable for post-processing and ii) availability of histopathological and molecular testing least for IDH1 and IDH2 mutations [2]. Images with non-correctable motion artefacts $(n=8)$ and corrupt source data $(n=18)$ were excluded. Finally, $333 \mathrm{pa}-$ tients were included in the analysis. Figure 1c contains the decision tree of the dataset stratified into different glioma cohorts.

\section{Histopathology}

All gliomas in the cohort were diagnosed according to the WHO 2016 classification scheme, complemented with molecular profiling for IDH1 and IDH2 mutations [2, 23-25]. The assessment of morphological features and immunostaining in the tumour samples was performed by neuropathologists in each institution. All IDH1R132H immune-negative gliomas were investigated by Sanger sequencing to identify out common tumour driver mutations of IDH1, IDH2, histone H3F3A, TERT promoter and BRAF genes [26, 27]. 1q/19p codeletion status, EGFR amplification and 10q loss were determined by using a qPCR-based copy number assay in a subset of the participating institutions.

\section{MR imaging}

The eligible patients were examined on either 1.5 T MR scanners $(n=186,56 \%)$ (Siemens SymphonyVision, Siemens Avanto, Siemens Healthineers, Erlangen, Germany; GE Discovery MR450, GE Healthcare, Chicago, USA; and Philips Achieva, Philips Medical Systems, Eindhoven, The Netherlands) or $3 \mathrm{~T}$ MR units ( $n=147,44 \%$ ) (Siemens Skyra, Siemens Allegra, Siemens 


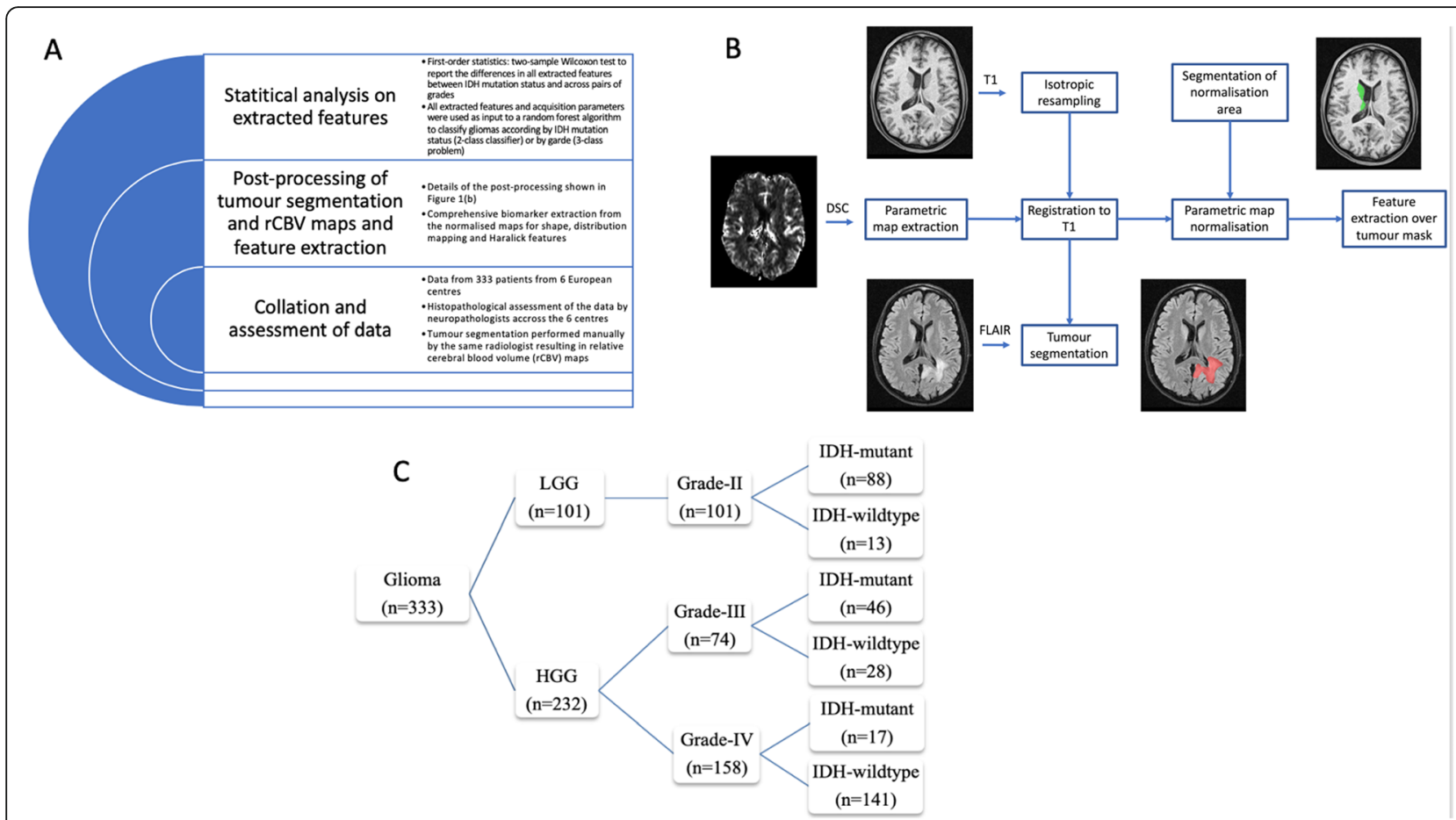

Fig. 1 a: Flow chart of the methodology of this analysis in this paper. $\mathbf{b}$ : Graphical illustration of the customised pipeline shows the cascade of processing starting from tumour segmentation on FLAIR series and ending in feature extraction over tumour mask. c: Decision tree illustrating the different cohorts of gliomas from the multicentre dataset stratified per WHO grades II, III and IV and also per IDH mutation status

TrioTim, Siemens Biograph_mMR, Siemens Healthineers, Erlangen, Germany; Philips Ingenia, Philips Medical Systems, Eindhoven, The Netherlands, $3 \mathrm{~T}$, Signa HDx, GE Healthcare, Waukesha, WI, USA).

\section{Tumour delineation and parametric modelling}

Tumour segmentation was performed manually by the same radiologist, who was blind to the immunohistopathological results, on axial T2 $(n=167,49.7 \%)$ or axial FLAIR $(n=169,51.3 \%)$ series using ITK-SNAP Version 3.6.0 [28] excluding large cystic or necrotic tumour components and large vessels in line with previous relevant literature. When available, 3D T2/FLAIR series were preferred to their 2D counterparts.

Relative cerebral blood volume (rCBV) maps were generated from the DSC -MRI raw data using dedicated commercially available postprocessing software (Olea Sphere, Version 3; Olea Medical Solutions, La Ciotat, France). Perfusion source images motion correction, temporal and spatial filtering was applied in all cases. Subsequently, the software automatically defined the arterial input function (AIF) for each individual based on all voxels throughout the time series using global clustering method as it was described by Mouridsen et al. [29]. Instead of the standard deconvolutional mathematical algorithms, a fully adaptive Bayesian scheme, which has been shown to be superior to the widely used classical approaches [30, 31], was chosen to derive the perfusion indices aiming at the most possibly accurate contrast agent leakage correction.

\section{Spatial and intensity normalisation}

The customised pipeline for the post-processing of the tumour segmentations and perfusion (rCBV) images is presented in Fig. 1b. The data was prepared for advanced analysis by isotropic resampling of the T1weighted images followed by registration, normalisation of the parametric rCBV maps, and biomarker extraction through distribution and texture features. Isotropic resampling to $1 \mathrm{~mm}^{3}$ voxels was facilitated using the NiftyReg open source software (https://sourceforge.net/ projects/niftyreg) to allow for the computation of rotational invariant textural features. The T2-weighted/ FLAIR images and $\mathrm{rCBV}$ maps were rigidly registered to the T1 isotropic image using NiftyReg [32] and trilinear interpolation. The resulting co-registered maps were visually inspected to evaluate the success of the registration step. At the third stage, automatic normalisation of the parametric maps was performed in order to mitigate inter-subject variability and therefore, enable comparison of statistical and textural features across subjects. At this step, the basal ganglia of both hemispheres were initially segmented using the Geodesic Information Flows framework (GIF) [33], a label fusion framework allowing 
for a local weighting of the information from a labelled database according to measures of local morphological similarities. The basal ganglia in the tumour-free hemisphere was used as a reference area for normalisation of the rCBV maps instead of healthy white matter in order to mitigate the risk of variability across the subjects due to white matter degeneration and vasculopathy. For tumours extending in the contralateral hemisphere, any tumour tissue was first removed from the reference region. Consequently, z-score maps over the tumour mask were derived with respect to the intensity distribution over the normalisation area.

\section{Feature extraction}

Comprehensive biomarker extraction was performed using the normalised parametric maps. To capture tumour shape, four shape features (volume, surface/volume ratio, non-compactness) were extracted for each tumour mass. For distribution mapping, 13 histogram features (mean, skewness, kurtosis, standard deviation, minimum, maximum, 1st, 5th, 25th, 50th (median), 75th, 95th and 99th percentiles) were extracted through all voxels in each segmented tumour. Finally, the 12 Haralick features [34] and including angular secondary moment (ASM), contrast, correlation, sum square, sum average, inverse difference moment (IDM), sum entropy, entropy, difference variance, sum variance, difference entropy, IMC1) for each tumour were also extracted.

\section{Statistical analysis}

All extracted intensity-based features were corrected for the influence of MR acquisition parameters on the extracted features was scrutinised by scanner manufacturer, magnetic field strength (1.5 T and $3 \mathrm{~T})$, TR $(\leq 1499 \mathrm{~ms}$ and $\geq 1500 \mathrm{~ms})$, TE $(25-44 \mathrm{~ms}$ and $45-55 \mathrm{~ms})$, FA $\left(90^{\circ}\right.$ and $\left.<90^{\circ}\right)$ slice thickness $(<5 \mathrm{~mm}$ and $\geq 5 \mathrm{~mm})$, and matrix size (matrix size $<128 \times 128$ and $\geq 128 \times 128$ ) and in plane resolution $(<=1,>1$ and $<=2,>=2)$. Following correction for these covariates, two-sample Wilcoxon test was used to report the differences in all extracted features between IDH mutation status and across pairs of grades. Results were considered to be significant if $p$-value $\leq 0.05$ and all statistical analysis was carried out using Stata v14 and python 3.7. Effect size difference was calculated using Cliff's Delta.

\section{Supervised learning from extracted features}

All extracted features and acquisition parameters were used as input to a random forest (RF) algorithm [35] to classify gliomas according to their IDH mutation status (IDH-mutant vs IDH-wildtype as a 2-class classifier) or according to their grade II, III and IV, as a 3-class problem. RF was chosen as the classifier based on the fact that it is simple to use as a collection of decision trees, is based on ensemble learning that allows the algorithm to learn accurately and does not require fine-tuning of parameters with the default parametrisation often leading to excellent performance.

For each task (IDH mutation or grade assessment) training was performed in a stratified two-fold crossvalidation setting with 250 repetitions to assess the value of rCBV-extracted features. Hyperparameters were optimised in a cross-validation setting leading to two different settings for the mutation task (200 trees, maximum depth of 10, minimum samples per leaf of 4) and the grade separation task (800 trees, minimum samples per leaf $=4$, maximum depth of 50). Error, computed as the difference between predicted and true classification was averaged over the 250 iterations and t-test over the error distribution were performed to assess the differences observed across acquisition parameters. Confusion matrices using the average classification were constructed to summarise the classification performance of the machine learning algorithm, from which the overall accuracy of classifying different gliomas cohorts, as well as overall sensitivity and specificity rates were calculated.

\section{Error introspection}

In order to have more insight into the cases of erroneous classification, features were compared for each category of misclassification (wild type classified as mutant, mutant as wild type, grade II as grade III etc) and their rightly classified counterpart to investigate if there was a consistency in the features leading to the misclassification and whether or not it was consisted demographic findings between said classes. Wilcoxon 2 samples tests were performed on the features corrected for acquisition parameters, age and gender as per the demographic analysis.

\section{Results \\ Demographic findings}

Overall, data included 333 glioma patients comprising 198 males and 135 females with a mean age 48.9 years (age range $20-81$ years). Of these, 101 were classified as grade II, 74 were grade III and 158 were grade IV while 151 were IDH-mutant and 182 were IDH-wildtype gliomas (Fig. 1c). Details on the demographics of the patient population, tumour location, histological grades and the molecular mutation status are contained in table S1 of Appendix A.

Effect size of acquisition parameters are presented with the value of the Cliff Delta in supplementary table S2. Shape, histogram and texture features for comparing gliomas by mutation status and across three different grades are shown in Table 1, with Cliff's Delta effects sizes presented pictorially in Fig. 2. 


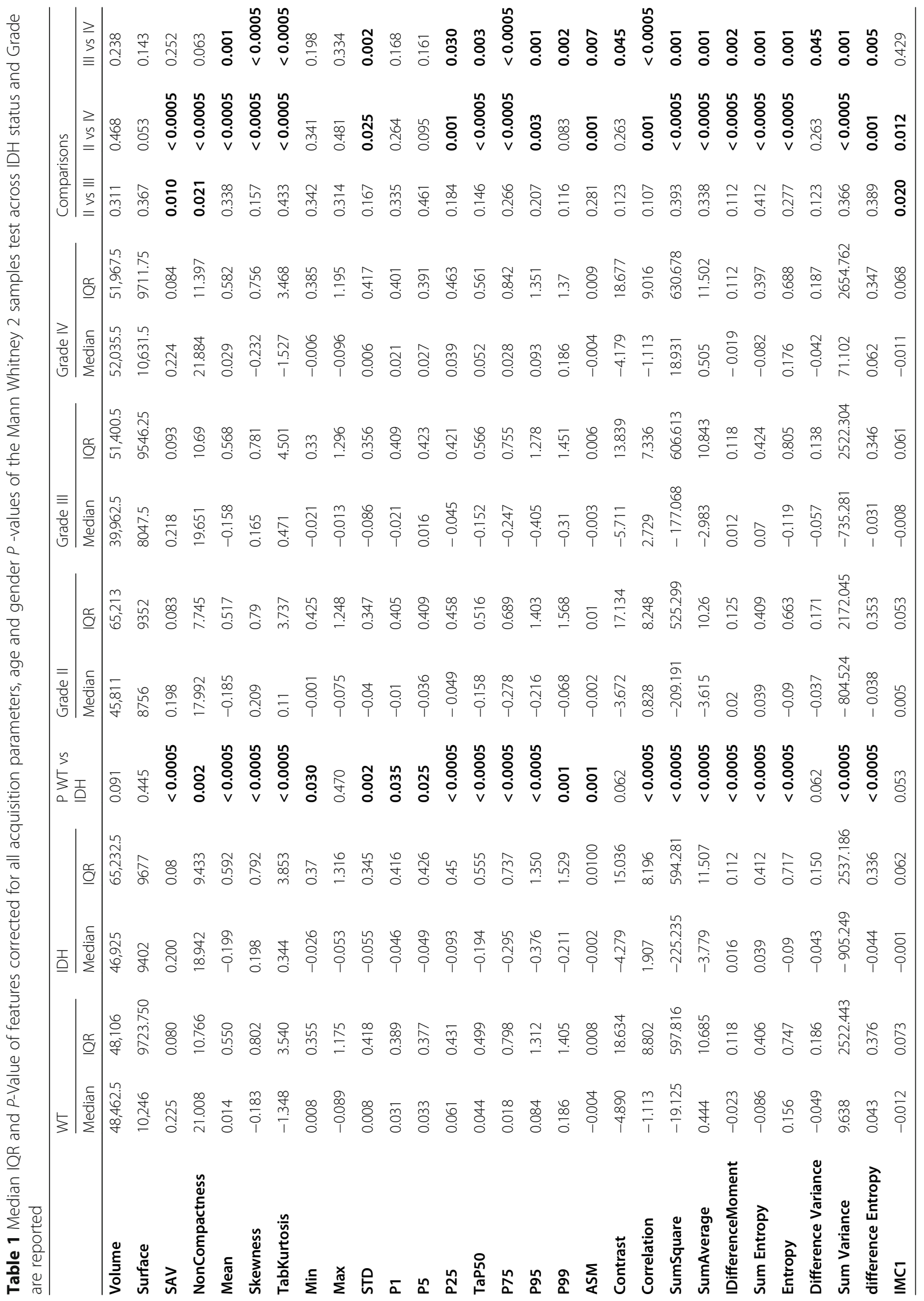




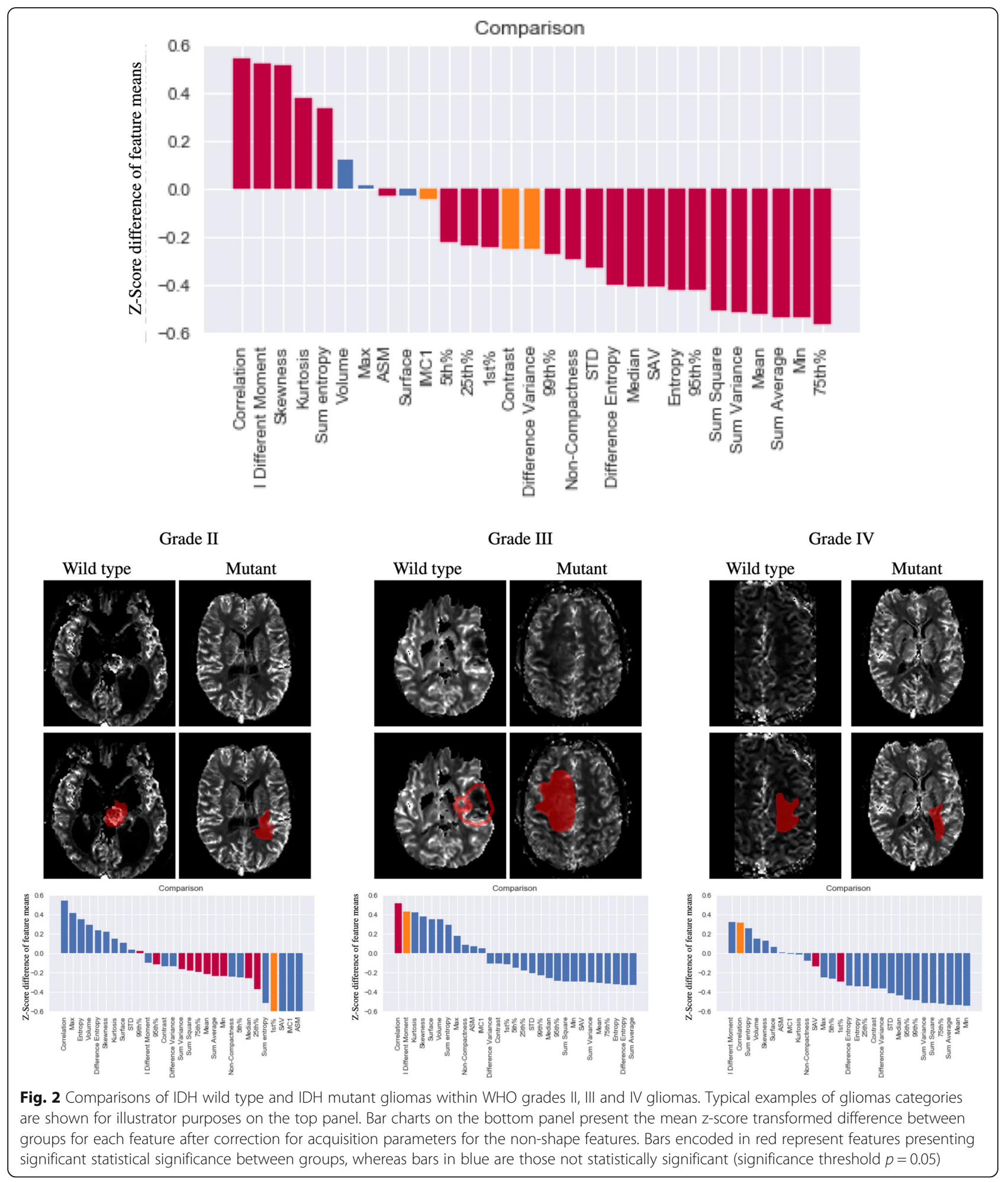

Glioma stratification for IDH mutation status

When comparing gliomas by mutation type, 23 out of the 29 extracted features were significantly different between IDH-wildtype and IDH-mutants (column 6 on
Table 1 and Fig. 2). Tumour surface to volume ratio (SAV) and measure of non-compactness were significantly lower in IDH-mutants compared to IDHwildtype, as were six rCBV histogram features, including 
mean, standard deviation,5th,25th,50th,75th, 95th and 99th percentiles. Skewness and kurtosis were higher in IDH-mutants compered to IDH-wildtype. Among the texture features, correlation and sum entropy were significantly higher in IDH-mutant tumours than in the IDH-wildtype $(p<0.0001)$, while sum square, sum average, sum variance, entropy and difference variance features were significantly lower $(p \leq 0.0001)$ in IDHmutant gliomas compared to IDH wildtype gliomas.

\section{Glioma stratification for WHO-grade}

The results of comparing gliomas by grade across WHO grades II, III and IV gliomas are shown in Fig. 3. When comparing gliomas grade III vs grade II, the surface to volume ratio shape feature, was significantly $(p=0.021)$ higher in grade III gliomas compared to grade II gliomas as was the non-compactness feature $(p=0.042)$ (Table 1 , column 13). The IMC1 measure was also found to be lower in grade III compared to grade II $(p=0.040)$.

A total of 20 features were different between grades III and IV (Fig. 3 and Table 1).The shape features did not differ between grades III and IV. Among the histogram features, 9 out of the 13 features were different with 6 (mean, STD, 25th, 50th, 75th, 95th and 99tth percentile) higher and skewness and kurtosis lower in grade IV compared to grade III gliomas. Finally, among the texture features 11 out of 12 features were different. Notably texture features related to appearance heterogeneity such as the entropy, sum square and sum variance were larger in grade IV than III, while measures of correlation and inverse difference momentum were lower.

Comparing gliomas grades IV with II, significant differences could be observed in most of the extracted features (20 out of 29). Of the shape features, SAV and non-compactness were higher, with non-compactness of greater degree, in gliomas grade IV compared to grade II. Five histogram features (mean, STD, 25th, 50th, 75th, 95th percentile) were higher while skewness and kurtosis were lower in grade IV compared to grade II gliomas. Finally, among the texture features, 10 out of 12 features, with only the contrast and the difference variance not presenting any statistically significant difference. Overall, comparisons between grade II and IV combined the differences observed between grade II and III and grade III and IV.

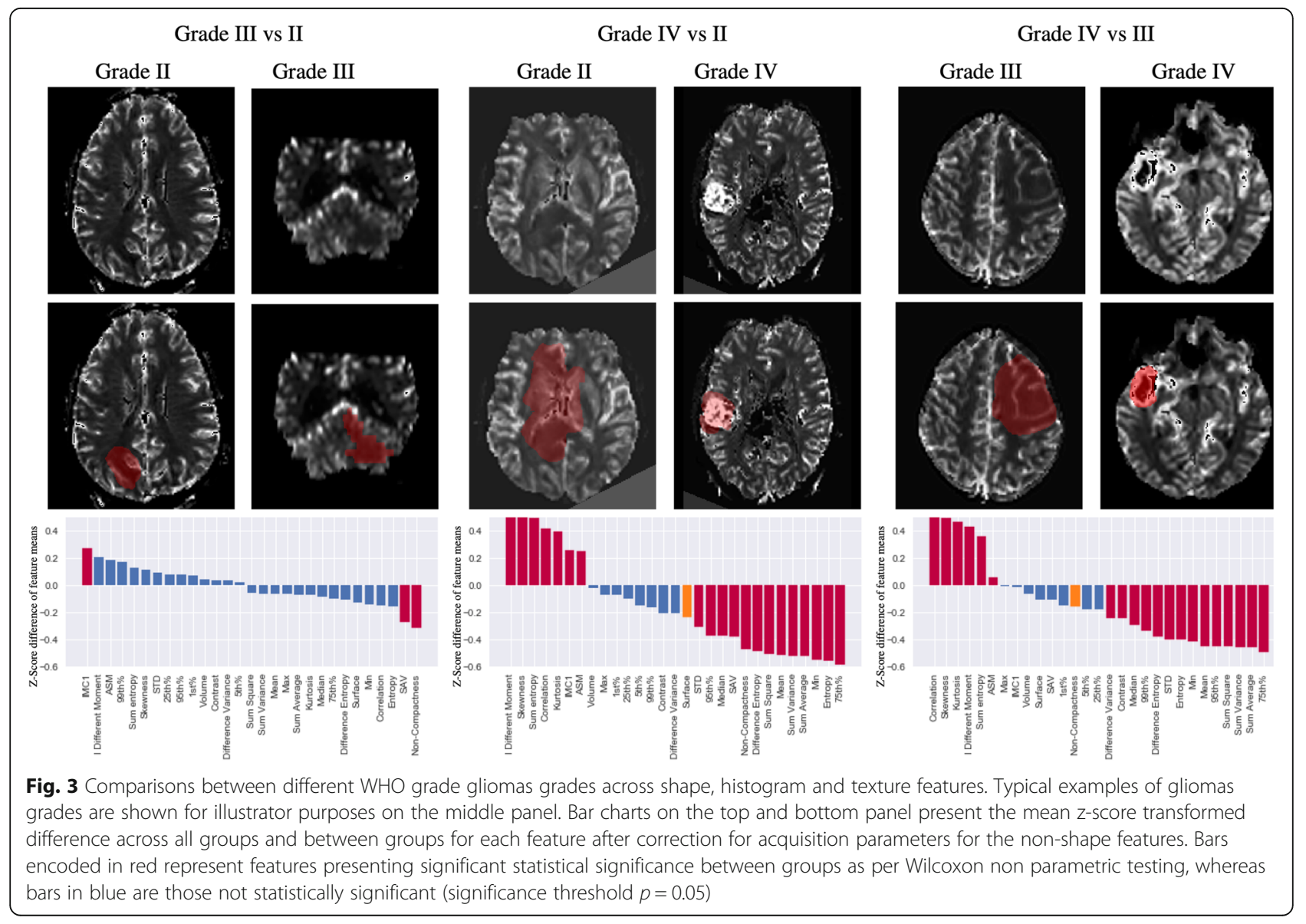




\section{Classification results}

The confusion matrices describing the accuracy of the random forest algorithm are contained in the supplementary table S5 and are depicted in Fig. 4a-b. The random forest algorithm correctly classified gliomas by IDH mutation status in $71 \%$ of the cases. This was slightly higher when we considered data from $1.5 \mathrm{~T}$ scanner only (77\% accuracy) while the accuracy dropped to $66 \%$ when we considered data from $3 \mathrm{~T}$ scanner only. Stratifying gliomas by IDH mutation status had an overall specificity of $77 \%$ and sensitivity of $65 \%$. When magnetic field strength was taken into consideration, at $1.5 \mathrm{~T}$ the specificity rate increased up to $83 \%$ with a sensitivity at $70 \%$ while the specificity was of $72 \%$ and the sensitivity of $60 \%$ at $3 \mathrm{~T}$. Overall, we obtained significant $(p=0.008)$ lower error rates of stratification at $1.5 \mathrm{~T}(0.25)$ in comparison to $3 \mathrm{~T}$ (0.35) (Table S3 and confusion matrices). Resolution appeared also to be associated with error, higher resolution leading to lower error rates $(0.196$ vs
$0.320 p=0.024$ for $<=1 \mathrm{~mm}$ vs between 1 and $2 \mathrm{~mm}$ ) (see Supplementary Table S2).

When classifying the gliomas by grade, $53 \%$ of included gliomas were classified correctly and $87 \%$ of the cases received grade classification with a distance less or equal to 1 . The accuracy of classifying gliomas by grade was higher using perfusion data obtained at $1.5 \mathrm{~T}$ magnetic field rather than at $3 \mathrm{~T}: 59$ and $46 \%$ respectively, $p=0.024$, with grade distance of less than 1 in 91 and $83 \%$ of the cases, respectively. Acquisition parameters had different effect with a larger error for lower resolution $(p=0.026)$, TR value $(p=0.009)$ with a larger error for TR below $1500 \mathrm{~ms}$; a flip angle value of 90 degrees was associated with higher error rates $(p=0.006)$.

\section{Introspection results}

When investigating in more details the misclassified cases (see Fig. 5), it appeared that the characteristics of these gliomas reflected the texture properties of the

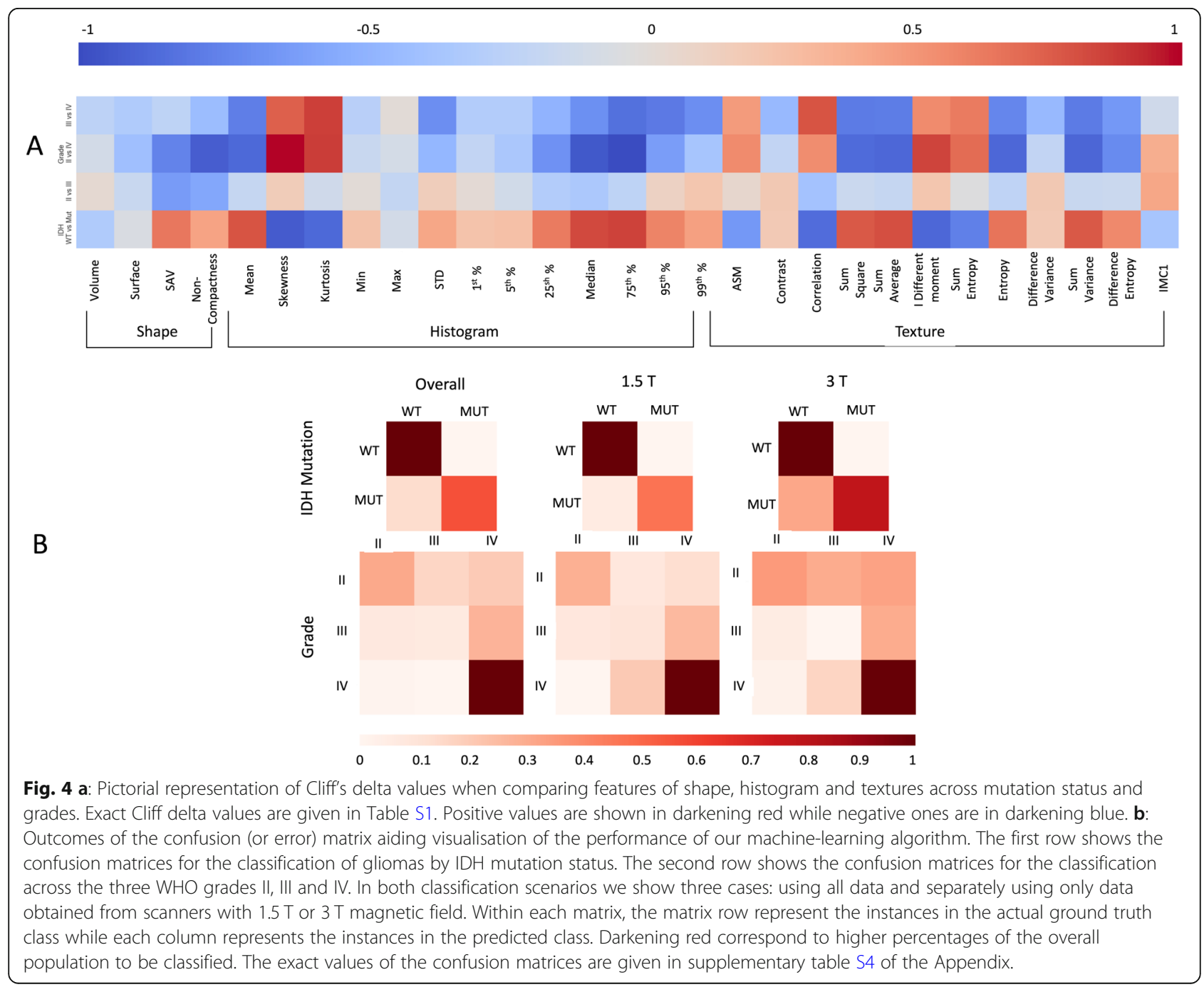




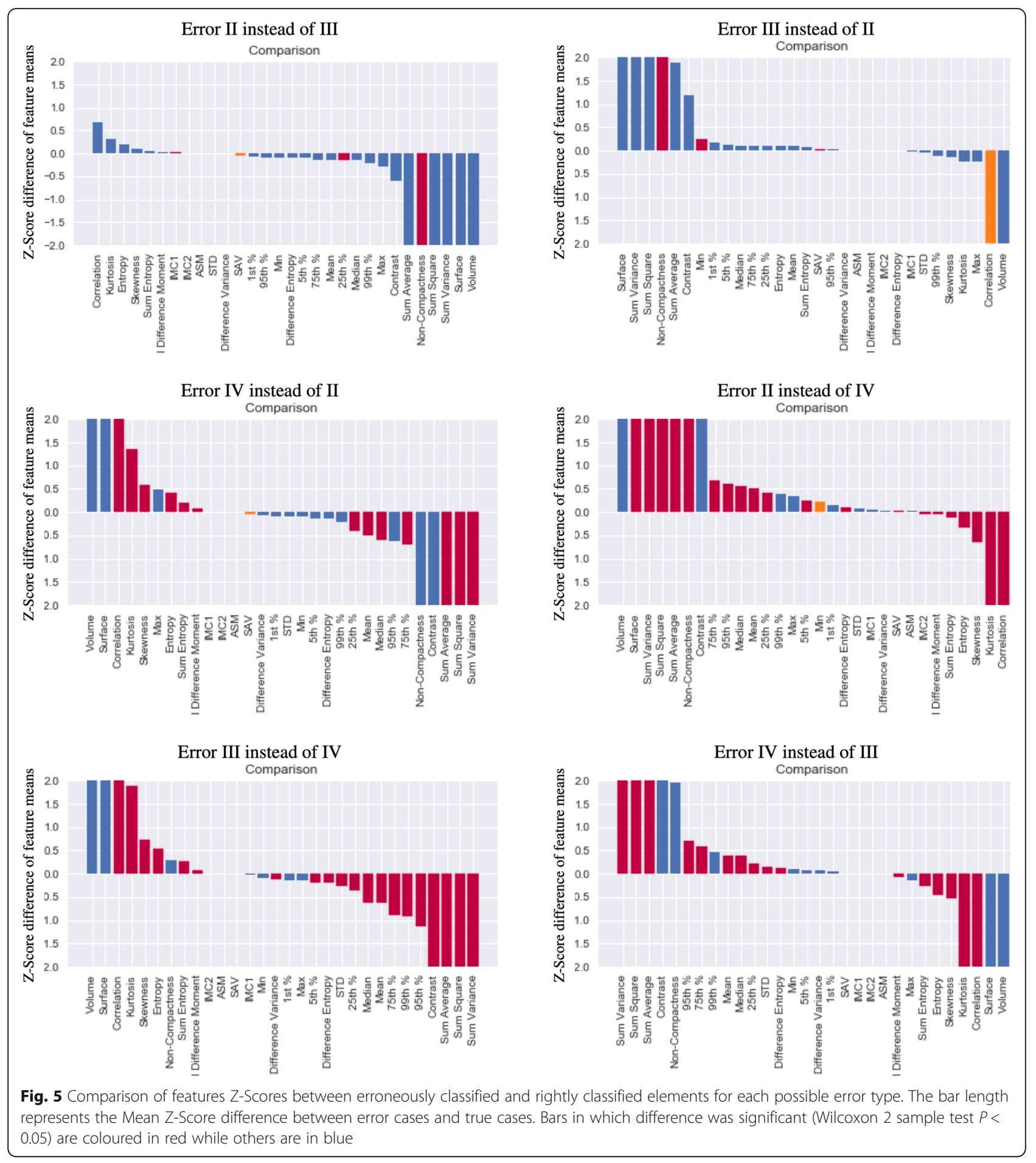

classes they were misclassified into both with respect to the mutation status or the grading. For instance, similarly to what was observed between grade II and IV, the cases wrongly classified as grade IV instead of grade II had a significantly lower compactness, with higher mean and kurtosis and lower skewness as well as lower correlation and higher entropy among others. Numerical mean of z-score differences for each of the possible error cases are reported in Supplementary table 4.

\section{Discussion}

Our results suggest that the application of machine learning strategies to DSC-MRI extracted features, has the potential to correctly classify gliomas from multi- 
center patients pool by IDH mutation status and WHO grades II, III and IV. Applying a random-forest algorithm, we accurately predicted IDH mutation status in $72 \%$ of the cases with an overall specificity of $77 \%$ and sensitivity of $65 \%$. On average, the algorithm classified $53 \%$ of the gliomas correctly into three histological grades II-IV, and with $87 \%$ the classification with an error distance of less than 1 .

The novelty of our work is that we show that even simple shape features can help in the distinction between grade II and higher grades. The measure of noncompactness was significantly higher in WHO grade III or grade IV compared to grade II, reflecting possibly higher infiltrative features in these tumours. Interestingly, this shape feature was not found to be significantly different between WHO grade IV and grade III groups. It must be noted that in the existing literature, histogram features have generally been favoured against shape descriptors [36-38]. Falk et al. have found that 90th percentile $\mathrm{rCBV}$ values were significantly lower in grade II gliomas than grade III (AUC $=0.77, p=0.04$ ), but they have also found the standard deviation of $\mathrm{rCBF}$ was the best discriminative feature with the highest AUC in the same setting (AUC $=0.80, p=0.02$ ). Our results do not confirm these findings, with histogram features only relevant markers of grade difference between grades IV and II or IV and III. Therefore, we suggest that shape descriptors, such as non-compactness and surface to volume ratio, have to be considered in the mapping of the composition of gliomas as they correlate well with histopathological findings. Perfusion texture features measuring the degree of intensity heterogeneity (such as the correlation or the sum of variance) appeared to be significantly different across the IDH mutation groups. Similar large number of markers of tissue heterogeneity were found to be different across a paired comparison of grades IV vs III and grades IV vs II. These observations are biologically relevant as they reflect heterogeneous microenvironment in high-grade gliomas, which is also consistent with diffusion studies [14, 39]. It can be postulated that texture features reflect the continuum of appearance evolution between grades but the results highlight the challenge of strictly separating grades III from the two extremes of the spectrum (grade II and grade IV).

The prediction rate in our study performed better (65\% of specificity, $79 \%$ sensitivity) than the one reported by Kickingereder et al. [8] in a single-centre trial despite the overall heterogeneous nature of our large cohort in terms of molecular subgroups and DSC acquisition protocols. Similar machine-assisted techniques for tumour grade prediction demonstrated accuracy between 73 and 85\% [40-43]. While our algorithm performance appears slightly inferior to the aforementioned results, we note that in all these studies patients are recruited from one centre, while we considered patients data from six centres. With multi-center data we expect higher variability and heterogeneity and hence anticipated lower accuracy. Using a different simulation approach might however improve accuracy. Citak-Er et al. [43] have proposed the use of a linear kernel support vector machine (SVM) with ten-fold cross-validation based on features extracted from conventional and advanced MRI data (DTI, DWI, DSC perfusion and MRSpectroscopy), and reported $73.3 \%$ of overall sensitivity from their multiparametric MR imaging. For the purpose of our study we chose random-forest algorithm as an established method for three classes or more classification. Future work would probably involve combining radiomics features from additional advanced MRI sequences so as to better identify required sequences for an optimised performance. With the recent rise of deeplearning techniques that do not rely on hand-crafted features, further exploration on this end would be required notably to assess the behaviour of such solutions in the identification of borderline cases, the associated uncertainty calibration as well as the assessment of robustness to acquisition changes.

Our error analysis was performed to better characterise the existing continuum between ordinal categories such as glioma grades. Analysis of the relationship between misclassifications and used features was strongly in agreement with previously well-known MRI shortcomings in tumour staging. For instance, some tumours incorrectly classified as WHO grade IV instead as grade III had significantly higher mean intensity in rCBV images. This highlights the inadequacy of first-order statistics to establish clear cut-off values regardless the sophistication of the DSC technique. This paradigm exemplifies the biological interpretability of machinelearning based radiomics compared to non-feature based methods and illustrates how it can be used to reevaluate categorical classifications and improve characterisation and understanding of the pathological continuum in glioma grades, in particular with emerging novel, validated molecular biomarkers, such as CDKN2A/B deletion in IDH-mutant astrocytomas, which have been suggested to form a new clinical risk group [3]. Such use of radiomics techniques parallels the findings of new biological definition of GBM subgroups described in the work from Rathore et al. [44].

Overall, this proof-of-concept study aimed to assess the diagnostic value of the DSC-MRI radiomics in treatment-naïve gliomas using multi-centre study design without a priori consensus on the acquisition parameters and settings (incl. MR field strength, sequence type, resolution, acquisition parameters). Different suggestions for sequence optimisation in DSC-PWI, especially across 
TE and FA, are available in the literature [30]. The large protocol variability observed in our cohort allowed us to investigate the effect of these key characteristics. Acquisition metrics were dichotomized based on existing literature [30, 45], and subsequently analysed prediction results across defined groups. Higher acquisition resolution was significantly associated with lower error rates. Error in grade prediction was more sensitive to acquisition parameters than the distinction between IDH wildtype and mutant. Additionally, lower field strength associated with lower variability in scanning protocols led to lower absolute errors $(p=0.02$ and $p=0.01$ for IDH and WHO grade respectively). Higher sensitivity and specificity rates with lower errors were achieved in the estimation of IDH mutation $(82,70 \%, 0.26$ at $1.5 \mathrm{~T}$, and $72,60 \%, 0.37$ at $3 \mathrm{~T}$, respectively), and better WHO grade predictions, exact or with a distance, at $1.5 \mathrm{~T}$ systems when compared to $3 \mathrm{~T}$. In fact, while higher spatial resolution and signal-to-noise ratio from higher magnetic fields may turn into an advantage in DSC imaging, which eventually leads decrease in the dose of contrast material and acquisitions time, protocol variability may increase image appearance heterogeneity. This result cannot be generalised in favour of the $1.5 \mathrm{~T}$ field strength scanners as this would need a controlled randomised study but it stresses the need for imaging acquisition guidelines to harness the potential of technological advances, such as higher MRI fields. Last but not least, partial volume effect caused by surrounding tissues in the point of arterial input function may also result in erroneous estimation of $\mathrm{rCBV}$.

The main strength of this work lies in both the multicentre design and, though limited for optimised learning approaches, still the largest cohort in the literature to utilise machine-learning based radiomics as a predictive diagnostic tool. Since the analyses were purposefully performed across perfusion data only, further studies are needed to assess a combined impact of a multimodal approach (e.g. DWI, T1-weighted MR perfusion, MR spectroscopy, PET) in the imaging phenotyping in gliomas, though this carries the risk of introducing further considerable variability.

This study has some limitations. Specifically, the small number of patients in some groups did not allow to form integrated molecular subgroups, akin to existing previous work. Therefore we could not undertake multiclass optimised learning classification according to the integrated histomolecular WHO classification scheme. Further data is continuously being gathered from the existing and new participating centres to extend the work in this direction and assess the generalisability of the proposed framework, while simultaneously exploring the optimal machine learning algorithm to use. Future work will explore the accuracy of contrasting different algorithms, such as supervised $\mathrm{V}$ unsupervised algorithms, when faced with heterogeneous dataset.

\section{Conclusions}

In conclusion, the findings underscore the satisfactory diagnostic contribution of DSC-MRI extracted higherorder features combined with machine-learning in the automated classification of grading and IDH mutation status of gliomas mitigating the high imaging heterogeneity. The promising results obtained through the proposed random forest radiomics-based framework, are an additional step towards the clinical translation of machine learning tools as diagnostic aid aiming at facilitating the implementation of tailored treatment based on precision imaging.

\section{Supplementary information}

Supplementary information accompanies this paper at https://doi.org/10. 1186/s12911-020-01163-5.

\section{Additional file 1: Table S1. Demographic details on the population. Table S2. Cliff's Delta obtained for the comparison of each feature (intensity and shape with respect to the different acquisition parameters. Table S3. Analysis of error and absolute errror in the prediction of IDH status and Grade according to acquisition parameters. Table S4. \\ Confusion matrix over different feld strengths for the mutation status prediction(top row) and the grade prediction(bottom row). Table S5. For all error types, mean z-score difference between the erroneously classified elements and the rightly classifed elements for each feature corrected for acquisition parameters. Figure S1. Representation of the acquisition pa- rameters repartition across the 333 used cases.}

\section{Abbreviations}

a-KG: a-ketoglutarate; 2-HG: D-2-hydroxyglutarate; AlF: Arterial input function; asm: Angular second moment; ATRX: Alpha thalassemia / mental retardation syndrome X- linked; BBB: Blood brain barrier; CBF: Cerebral blood flow; CBV: Cerebral blood volume; cWM: Contralateral white matter; DSCMRI: Dynamic susceptibility contrast-enhanced MRI; EGFR: Epidermal growth factor receptor; GBM: Glioblastoma multiforme; Gd: Gadolinium; IDH: Isocitrate dehydrogenase; imc: Information measures of correlation; $\mathrm{k}_{2}$ : Constant of contrast agent permeability; MGMT: O6 - methylguanineDNA - methyltransferase; rCBV: Relative cerebral blood volume; ROI: Region of interest; SAV: Sum average; SVM: Support vector machine;

TERT: Telomerase reverse transcriptase

\section{Acknowledgments}

Not applicable.

\section{Authors' contributions}

CHS, JPG, ES, MJC and SB2 conceived the study. ES undertook the preprocessing of the images and extracted the features for statistical analysis. CHS and JPG undertook the statistical analysis. JPG, CHS and ES drafted the manuscript with input from SB1, SB2 and MJC. VKK, GS, FBP, CG, KSP, JA, MVS, MN, ARC, AA, SG, AK, NA, GMC, VR, LU, AE, EFC,EG,EK supplied the data for this study and reviewed the manuscript. DR, JG and TB reviewed and and edited later versions of the manuscript. All co-authors read and approved the submitted version of the manuscript.

\section{Funding}

CS is supported by an Alzheimer's Society Junior Fellowship (AS-JF-17-011). MJC is supported by Wellcome/EPSRC Centre for Medical Engineering (WT203148/Z/16/Z) and Wellcome Flagship Programme (WT213038/Z/18/Z). JPG's work was funded by the National Institute for Health Research (NIHR) Applied Health Research and Care North Thames at Barts Health NHS Trust. Part of the study was funded by the National Institute for Health Research to 
UCLH Biomedical research centre (BRC399/NS/RB/101410). SBr is also supported by the Department of Health's NIHR Biomedical Research Centre's funding scheme. The views expressed are those of the authors and not necessarily those of the NHS, the NIHR or the Department of Health and Social Care. The funder had no role in this study.

\section{Availability of data and materials}

The datasets used and analysed during the current study, and the machine learning algorithm are available from the corresponding author on reasonable request.

\section{Ethics approval and consent to participate}

Informed consent was deemed unnecessary due to the retrospective nature of the study and according to the national ethics regulations for medical research. The ethics approval protocol was submitted to the University College London / University College London Hospitals Joint Research Office (Reference 213920) and the assigned North West - Liverpool Central Research Ethics Committee approved the study (reference number: 18/NW 0395). In addition bilateral data transfer agreements reviewed by the University College London Hospitals Joint Research Office and the counterpart research committees were put in place with each contributing institution. All individual data was anonymised and only collated data on perfusion parameters was seen by statisticians.

\section{Consent for publication}

Not applicable.

\section{Competing interests}

The authors declare that they have no competing interests.

\section{Author details}

'Department of Biomedical Engineering, School of Biomedical Engineering \& Imaging Sciences, King's College, London, UK. ${ }^{2}$ Dementia Research Centre, Institute of Neurology, University College London, London, UK. ${ }^{3}$ Department of Medical Physics and Biomedical Engineering, University College London, London, UK. ${ }^{4}$ Department of Applied Health Research, Institute of Epidemiology \& Health Care, University College London, London, UK. ${ }^{5}$ Institute for Global Health, University College London, London, UK. ${ }^{6}$ The Queen's College, Oxford University, Oxford, UK. ${ }^{7}$ Department of Neuroradiology, The National Hospital for Neurology and Neurosurgery, University College London NHS Foundation Trust, London, UK. ${ }^{8}$ Division of Neuropathology, UCL Queen Square Institute of Neurology, London, UK. ${ }^{9}$ Department of Advanced Imaging Modalities, MRI Unit, General Anti-Cancer and Oncological Hospital of Athens "St. Savvas", Athens, Greece.

${ }^{10}$ Department of Neurosurgery, General Hospital Evangelismos, Medical School, University of Athens, Athens, Greece. ${ }^{11}$ Neuroradiology, Department of Diagnostics and Pathology, Verona University Hospital, Verona, Italy. ${ }^{12}$ Neuropathology, Department of Diagnostics and Pathology, Verona University Hospital, Verona, Italy. ${ }^{13}$ Department of Neuroradiology, University Medical Centre, Ljubljana, Slovenia. ${ }^{14}$ Department of Radiology, Faculty of Medicine, University of Ljubljana, Ljubljana, Slovenia. ${ }^{15}$ Department of Radiology and Radiological Science, Medical University of South Carolina, Charleston, SC, USA. ${ }^{16}$ Grenoble Institute of Neurosciences, INSERM, University Grenoble Alpes, Grenoble, France. ${ }^{17}$ Department of Neuroradiology, San Raffaele Hospital, Vita-Salute San Raffaele University, Milan, Italy. ${ }^{18}$ Department of Advanced Biomedical Sciences, Diagnostic Imaging Section, University of Naples Federico II, Naples, Italy. ${ }^{19}$ Department of Advanced Biomedical Sciences, Pathology Section, University of Naples Federico II, Naples, Italy. ${ }^{20}$ Department of Radiology, School of Health Sciences, Faculty of Medicine, University of Thessaly, Larisa, Greece. ${ }^{21}$ Image Analysis Group, London, UK. ${ }^{22}$ Olea Medical, La Ciotat, France. ${ }^{23}$ Department of Brain Repair and Rehabilitation, Queen Square Institute of Neurology, UCL, London, UK.

Received: 21 October 2019 Accepted: 24 June 2020

Published online: 06 July 2020

\section{References}

1. Yang M, Soga T, Oncometabolites PPJ. Linking altered metabolism with cancer. J Clin Invest. 2013;123(9):3652-8.
2. Louis DN, Perry A, Reifenberger G, von Deimling A, Figarella-Branger D, Cavenee WK. And others. The 2016 World Health Organization classification of tumors of the central nervous system: a summary. Acta Neuropathol. 2016;131(6):803-20.

3. Shirahata M, Ono T, Stichel D, et al. Novel, improved grading system(s) for IDH-mutant astrocytic gliomas. Acta Neuropathol. 2018;136(1):153-66.

4. Chamberlain MC, Murovic JA, Levin VA. Absence of contrast enhancement on CT brain scans of patients with supratentorial malignant gliomas. Neurology. 1988;38(9):1371

5. Scott JN, Pma B, Sevick RJ, Rewcastle NB, PA. F. How Often Are Nonenhancing Supratentorial Gliomas Malignant? A Population Study. Neurology. 2002;59(6):947-9.

6. Santarosa C, Castellano A, Conte GM, et al. Dynamic contrast-enhanced and dynamic susceptibility contrast perfusion MR imaging for Glioma grading: preliminary comparison of vessel compartment and permeability parameters using hotspot and histogram analysis. Eur J Radiol. 2016;85(6): 1147-56.

7. Anzalone N, Castellano A, Cadioli M, et al. Brain Gliomas: Multicenter Standardized Assessment of Dynamic Contrast-enhanced and Dynamic Susceptibility Contrast MR Images. Radiology. 2018;1703:62.

8. Kickingereder $\mathrm{P}$, Bonekamp D, Nowosielski $\mathrm{M}$, et al. Radiogenomics of Glioblastoma: machine learning-based classification of molecular characteristics by using multiparametric and multiregional MR imaging features. Radiology. 2016;281(3):907-18.

9. Hu LS, Kelm Z, Korfiatis $P$, et al. Impact of software modeling on the accuracy of perfusion MRI in Glioma. AJNR Am J Neuroradiol. 2015;36(12):2242-9.

10. Kelm ZS, Korfiatis PD, Lingineni RK, et al. Variability and accuracy of different software packages for dynamic susceptibility contrast magnetic resonance imaging for distinguishing glioblastoma progression from pseudoprogression. J Med Imaging. 2015;2:2.

11. Conte GM, Castellano A, Altabella L, et al. Reproducibility of dynamic contrast-enhanced MRI and dynamic susceptibility contrast MRI in the study of brain gliomas: a comparison of data obtained using different commercial software. Radiol Med. 2017;122(4):294-302.

12. Welker K, Boxerman J, Kalnin A, et al. ASFNR recommendations for clinical performance of MR dynamic susceptibility contrast perfusion imaging of the brain. AJNR Am J Neuroradiol. 2015;36(6):E41-51.

13. Brynolfsson $P$, Nilsson $D$, Henriksson $R$, et al. ADC texture-an imaging biomarker for high-grade glioma? Med Phys. 2014;41(10):101903. https://doi. org/10.1118/1.4894812.

14. Bisdas S, Shen H, Thust S, et al. Texture analysis- and support vector machine-assisted diffusional kurtosis imaging may allow in vivo gliomas grading and IDH-mutation status prediction: a preliminary study. Sci Rep. 2018:8:1.

15. Chaddad A, Zinn PO, Colen RR. Quantitative texture analysis for Glioblastoma phenotypes discrimination. In: 2014 International conference on control, decision and information technologies (CoDIT), vol. 2014: IEEE. p. 605-8. https://doi.org/10.1109/CoDIT.2014.6996964.

16. Yang D, Rao G, Martinez J, Veeraraghavan A, Rao A. Evaluation of tumorderived MRI-texture features for discrimination of molecular subtypes and prediction of 12-month survival status in glioblastoma. Med Phys. 2015; 42(11):6725-35. https://doi.org/10.1118/1.4934373.

17. McGarry SD, Hurrell SL, Kaczmarowski AL, et al. Magnetic Resonance Imaging-Based Radiomic Profiles Predict Patient Prognosis in Newly Diagnosed Glioblastoma Before Therapy. Tomography. 2016;2(3):223. https:// doi.org/10.18383/J.TOM.2016.00250.

18. Kickingereder P, Gotz M, Muschelli J, et al. Large-scale Radiomic profiling of recurrent Glioblastoma identifies an imaging predictor for stratifying antiAngiogenic treatment response. Clin Cancer Res. 2016;22(23):5765-71. https://doi.org/10.1158/1078-0432.CCR-16-0702.

19. Grossmann P, Narayan V, Chang K, et al. Quantitative imaging biomarkers for risk stratification of patients with recurrent glioblastoma treated with bevacizumab. Neuro-Oncology. 2017;19(12):1688-97. https://doi.org/10. 1093/neuonc/nox092.

20. Ertosun MG, Rubin DL. Automated grading of Gliomas using deep learning in digital pathology images: a modular approach with ensemble of convolutional neural networks. AMIA. Annu Symp proceedings AMIA Symp. 2015;2015:1899-908.

21. Chang P, Grinband J, Weinberg BD, et al. Deep-learning convolutional neural networks accurately classify genetic mutations in Gliomas. Am J Neuroradiol. 2018;39(7):1201-7. https://doi.org/10.3174/AJNR.A5667. 
22. Lu CF, Hsu FT, Hsieh KL, et al. Machine learning-based Radiomics for molecular subtyping of Gliomas. Clin Cancer Res. 2018;24(18):4429-36.

23. Hartmann C, Meyer J, Balss J, et al. Type and frequency of IDH1 and IDH2 mutations are related to astrocytic and oligodendroglial differentiation and age: a study of 1,010 diffuse gliomas. Acta Neuropathol. 2009;118(4):469-74.

24. Louis DN, Perry A, Burger P, Ellison DW, Reifenberger G, von Deimling A. And others. International society of neuropathology--Haarlem consensus guidelines for nervous system tumor classification and grading. Brain Pathol. 2014;24(5):429-35.

25. Balss J, Meyer J, Mueller W, Korshunov A, Hartmann C, von Deimling A. Analysis of the IDH1 codon 132 mutation in brain tumors. Acta Neuropathol. 2008;116(6):597-602.

26. Brandner S, von Deimling A. Diagnostic. Prognostic and predictive relevance of molecular markers in gliomas. Neuropathol Appl Neurobiol. 2015;41(6): 694-720.

27. Jaunmuktane Z, Capper D, Dtw J, et al. Methylation array profiling of adult brain tumours: diagnostic outcomes in a large, single Centre. Acta Neuropathol Commun. 2019;7:1.

28. Yushkevich PA, Piven J, Hazlett HC, et al. User-guided 3D active contour segmentation of anatomical structures: significantly improved efficiency and reliability. Neuroimage. 2006;31(3):1116-28.

29. Mouridsen K, Friston K, Hjort N, Gyldensted L, Østergaard L, Kiebel S. Bayesian estimation of cerebral perfusion using a physiological model of microvasculature. Neuroimage. 2006;33(2):570-9. https://doi.org/10.1016/j. neuroimage.2006.06.015.

30. Boxerman $J L$, Paulson ES, Prah MA, Schmainda KM. The effect of pulse sequence parameters and contrast agent dose on percentage signal recovery in DSC-MRI: implications for clinical applications. AJNR Am J Neuroradiol. 2013;34(7):1364-9.

31. Boxerman JL, Schmainda KM, Weisskoff RM. Relative cerebral blood volume maps corrected for contrast agent extravasation significantly correlate with glioma tumor grade, whereas uncorrected maps do not. AJNR Am J Neuroradiol. 2006;34(7):1364-9.

32. Modat M, Cash DM, Daga P, Winston GP, Duncan JS, Ourselin S. Global image registration using a symmetric block-matching approach. J Med Imaging. 2014;1(2):024003.

33. Cardoso MJ, Modat M, Wolz R, et al. Geodesic information flows: spatiallyvariant graphs and their application to segmentation and fusion. IEEE Trans Med Imaging. 2015;34(9):1976-88. https://doi.org/10.1109/TMI.2015.2418298.

34. Haralick RM, Shanmugam K, Dinstein I. Textural features for image classification. IEEE Trans Syst Man Cybern Part B. 1973;SMC-3(6):61021.

35. Breiman L. Statistical modeling: the two cultures. Stat Sci. 2001;16(3):199-215.

36. Catalaa I, Henry R, Dillon WP, et al. Perfusion, diffusion and spectroscopy values in newly diagnosed cerebral gliomas. NMR Biomed. 2006;19(4):463-75.

37. Kim H, Choi SH, Kim JH, et al. Gliomas: application of cumulative histogram analysis of normalized cerebral blood volume on 3 T MRI to tumor grading. PLoS One. 2013;8:5.

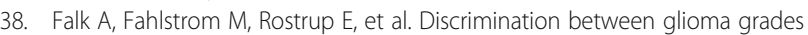
II and III in suspected low-grade gliomas using dynamic contrast-enhanced and dynamic susceptibility contrast perfusion MR imaging: a histogram analysis approach. Neuroradiology. 2014;56(12):1031-8.

39. Hempel JM, Schittenhelm J, Bisdas S, et al. In vivo assessment of tumor heterogeneity in WHO 2016 glioma grades using diffusion kurtosis imaging: diagnostic performance and improvement of feasibility in routine clinical practice. J Neuroradiol. 2018;45(1):32-40.

40. Zöllner FG, Emblem KE, Schad LR. Support vector machines in DSC-based glioma imaging: suggestions for optimal characterization. Magn Reson Med. 2010;64:1230-6.

41. Emblem KE, Due-Tonnessen P, Hald JK, Bjornerud A, Pinho MC, Scheie D, et al. Machine learning in preoperative glioma MRI: survival associations by perfusion-based support vector machine outperforms traditional MRI. J Magn Reson Imaging. 2014;40(1):47-54.

42. Emblem KE, Pinho MC, Zollner FG, Due-Tonnessen P, Hald JK, Schad LR, et al. A generic support vector machine model for preoperative glioma survival associations. Radiology. 2015;275(1):228-34.

43. Citak-Er F, Firat Z, Kovanlikaya I, Ture U, Ozturk-Isik E. Machine-learning in grading of gliomas based on multi-parametric magnetic resonance imaging at 3T. Comput Biol Med. 2018;99:154-60.

44. Rathore S, Akbari H, Rozycki M, et al. Radiomic MRI signature reveals three distinct subtypes of glioblastoma with different clinical and molecular characteristics, offering prognostic value beyond IDH1. Sci Rep. 2018;8(1): 5087. https://doi.org/10.1038/s41598-018-22739-2.

45. Boxerman $J$, Rosen BR, Weisskoff RM. Signal-to-noise analysis of cerebral blood volume maps from dynamic NMR imaging studies. J Magn Reson Imaging. 1997;7(3):528-37.

\section{Publisher's Note}

Springer Nature remains neutral with regard to jurisdictional claims in published maps and institutional affiliations.
Ready to submit your research? Choose BMC and benefit from:

- fast, convenient online submission

- thorough peer review by experienced researchers in your field

- rapid publication on acceptance

- support for research data, including large and complex data types

- gold Open Access which fosters wider collaboration and increased citations

- maximum visibility for your research: over $100 \mathrm{M}$ website views per year

At BMC, research is always in progress.

Learn more biomedcentral.com/submissions 